\title{
Association between Temporomandibular Disorder and Masticatory Muscle Weakness: A Case report
}

\author{
Ji Hoo Kim ${ }^{1}$, Hyun-Jeong Park ${ }^{1,2}$, Ji-Won Ryu ${ }^{1,2}$ \\ 1 Department of Oral Medicine, Chosun University Dental Hopital, Gwangju, Korea \\ ${ }^{2}$ Department of Oral Medicine, School of Dentistry, Chosun University, Gwangju, Korea
}

Received November 25, 2021

Revised December 6, 2021

Accepted December 7, 2021

\section{Correspondence to:}

Ji-Won Ryu

Department of Oral Medicine, School of Dentistry, Chosun University, 309 Pilmundaero, Dong-gu, Gwangju 61452, Korea

Tel: +82-62-220-3897

Fax: +82-62-234-2119

E-mail:dentian@chosun.ac.kr

https://orcid.org/0000-0002-5586-8195

This study was supported by research fund from Chosun University Dental Hospital, 2019.
The masticatory muscle disorder is the most common problem that patients with temporomandibular disorder often complain. For such complaints, treatment is directed towards reducing hyperactivity of muscles or effects of the central nervous system. However, if nonspecific occlusal change or pain persists, it is necessary to consider that muscle weakness might be the cause of the persistence of temporomandibular disorder. Stabilization of occlusion and improvement of the pain symptoms were achieved in both cases through the chewing gum exercise. This exercise may enable masticatory movements done in normal function by using muscle engram and achieve reinforcement of the masticatory muscles with balanced, simultaneous contacts of the teeth. In addition, it may be a viable method for treating temporomandibular disorders that do not respond well to conventional mandibular stabilization therapies.

Key Words: Chewing gum; Exercise; Muscle weakness; Temporomandibular disorder; Ultrasonography

\section{INTRODUCTION}

Temporomandibular disorders (TMDs) may arise from a variety of causes, including occlusal status, trauma, emotional stress, inflow of deep pain, and abnormal functional activity [1]. It is suspected that symptoms of TMDs may appear when stimuli affecting the masticatory function exceed the range of physiological tolerance during the normal functions of the masticatory system [2].

When patients present with symptoms related to TMDs, especially pain, possible causes related to hyperactivity of the muscles of the masticatory system are evaluated and various treatment regimens are implemented to promote the stabilization of the masticatory system [2]. On the other hand, chronic pain may cause muscle weakness via changes in the intramuscular system. If the mandibular movement is reduced to avoid such pain, it may further lead to shortened and atrophied muscle length, which may ironically elicit pain again. Therefore, treatment progresses of patients with TMD related to the weakening of the masticatory muscles - especially the masseter muscle - are investigated in this study reviewing two cases, and diagnosis and treatment options are discussed.

\section{CASE REPORT}

The protocol of this study was approved by the Institutional Review Board (IRB) of Chosun University Dental Hospital (IRB no. CUDHIRB 2106 005).

\section{Case 1}

A 39-year-old male visited to the department of Oral Medicine in Chosun University Dental Hospital with the chief complaints of having "pain in the left mandible and

Copyright (c) 2021 Korean Academy of Orofacial Pain and Oral Medicine

(c) This is an open-access article distributed under the terms of the Creative Commons Attribution Non-Commercial License (http://creativecommons.org/licenses/by-nc/4.0/), which permits unrestricted non-commercial use, distribution, and reproduction in any medium, provided the original work is properly cited. 
the zygomatic area on both sides after coming down from a pull-up while pushing down on the chin 3 weeks ago" as well as "constant tingling and unnatural feeling when chewing food".

Clinical examination of this patient revealed active mouth opening of $45 \mathrm{~mm}$ without pain and opening limitations. However, tenderness in the left and the right masseter muscle areas was reported when the masticatory muscles were palpated. The overjet and overbite were measured 0 $\mathrm{mm}$ each, and the midline was deviated $2 \mathrm{~mm}$ to the right side. Using an 8- $\mu \mathrm{m}$ thick shimstock paper to study the intercuspal position showed occlusion of \#17-13, 11 on \#41, 43-47, and \#21-27 on \#37-31. Panoramic, transcranial, lateral anterior head, and posterior anterior head radiographs showed no remarkable lesions in both TMJs, teeth and the surrounding tissues. There was no restriction of the anterior range of motion of the mandible. The patient was diagnosed with myalgia of the masseter muscles on both sides from the results of the examination. The patient received physical therapy, self-treatment instruction, and the following medications: anti-inflammatory drugs (aceclofenac 100$\mathrm{mg}$ bis in die [bid]) and tricyclic antidepressants (amitriptyline 10-mg hora somni [hs]).

Although the intensity of the pain decreased after followup appointments, symptoms such as stiffness, tingling, and lumpiness in zygomatic areas and masseter muscles on both sides continued to persist or even worsened despite multiple treatment attempts. Because the patient did not comply with the recommendation to wear an occlusal appliance, only physical therapy and intermittent drug therapy could be performed to reduce muscle hyperactivity and stabilize the mandible. However, the symptoms persisted without any relief during monitoring period.

About 4 months after starting the initial set of treatments, the patient complained of having severe pain and sudden change in occlusion. Clinical examination revealed that the overbite decreased to $-1 \mathrm{~mm}$, and the shimstock paper showed anterior open bite and occlusion of molars only on both sides (Fig. 1). Cone beam computed tomography (CBCT) of the TMJ and lateral radiographs were taken (Fig. 2), which revealed no remarkable bone changes. The patient did not visit the hospital for about 5 months, and then he came back at about 9 months after the initial treatment, showing no improvements in symptoms. The patient reported he visited another university hospital and took magnetic resonance imaging (MRI) of the TMJ, in which the position of the articular disks in both TMJ areas were normal, and no muscle inflammation was observed. The intercuspal position also remained unchanged from 5 months ago. The patient allegedly went through similar kinds of treatments in the other hospital and wondered if there was another treatment option. There was no significant difference in the range of mouth opening upon clinical examination, but the patient complained that he needed to use more force to open his mouth. For further investigation, the patient was instructed to clench his teeth to evaluate contractility of his
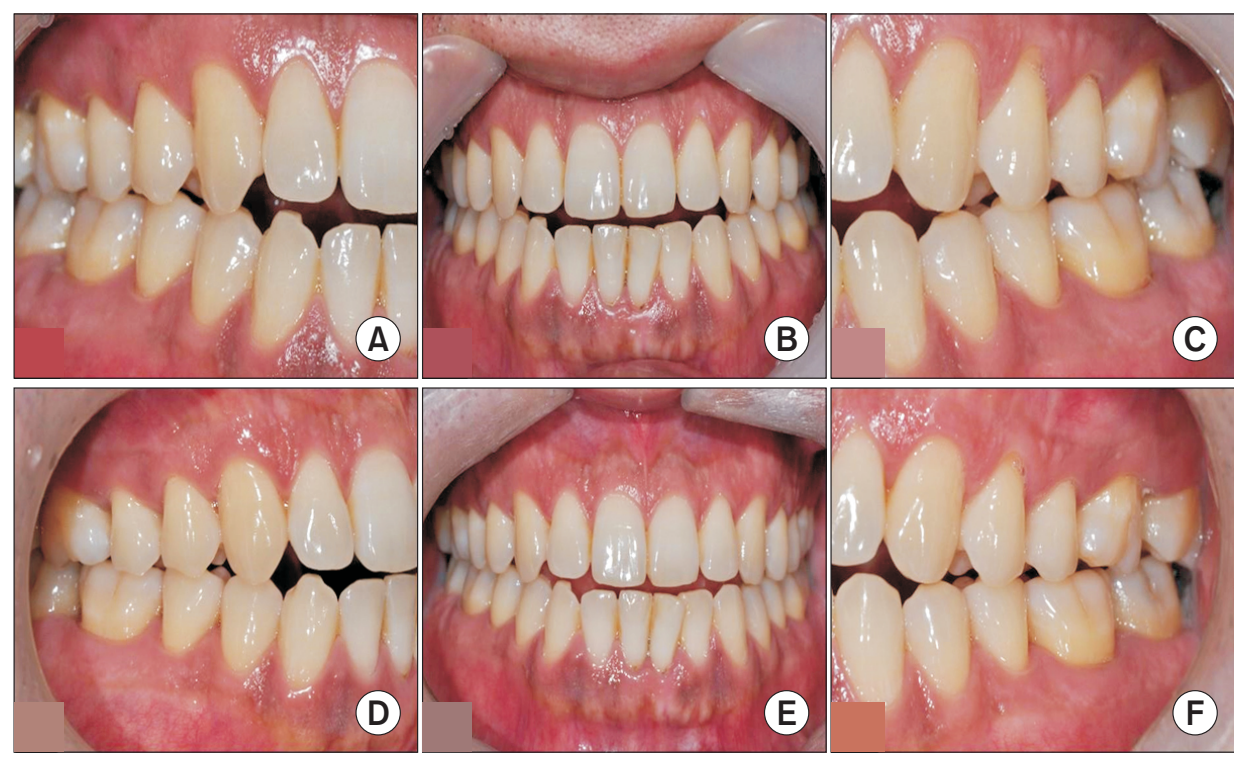

C

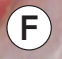

Fig. 1. Occlusal photo of case 1. (A C) 4 months later, $(D-F) 1$ year and 4 months later. 

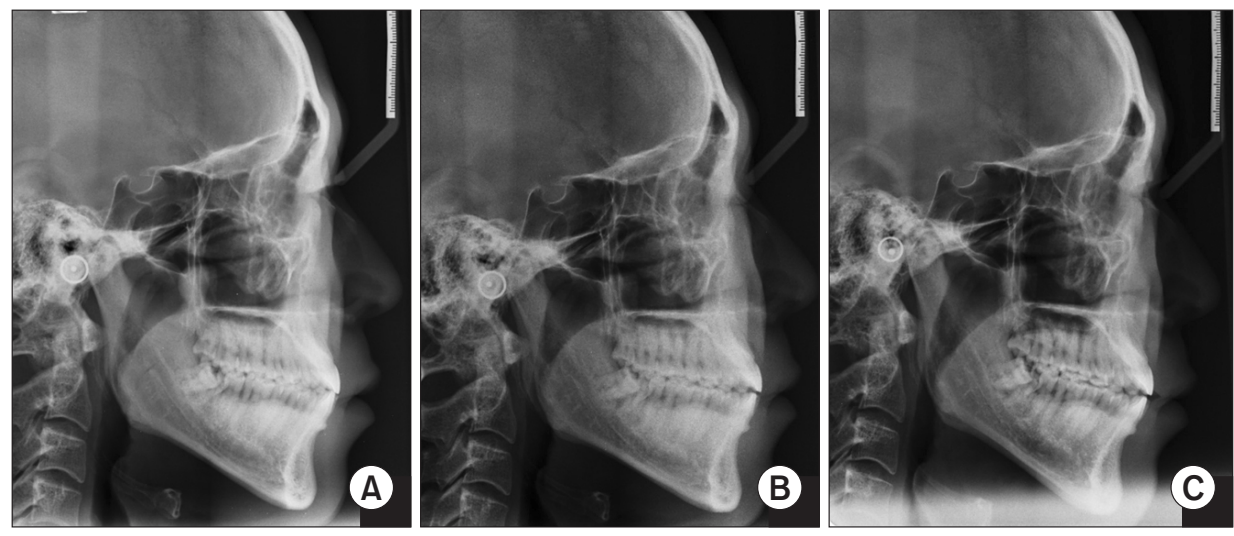

Fig. 2. Lateral cephalometric radiography of case 1. (A) First visit, (B) 4 months later, (C) 1 year and 4 months later. masticatory muscles, which showed no contraction of both masseter muscles. Therefore, a muscle weakness post trauma was regarded as a major factor that was contributing to his symptoms, and the patient was instructed to perform bilateral gum chewing as a measure to increase muscle strength. The progress of the symptoms was observed for 6 months, during which the patient continued to perform bilateral gum chewing to increase muscle strength and received anti-inflammatory drugs as needed to control the pain if it worsened. As results, there was improvement in the pain symptoms and occlusion, although not the same as the first visit, and the overbite was reduced to $-0.5 \mathrm{~mm}$ (Fig. 1). The patient still reported unnatural feeling during mouth opening, but it gradually improved and eventually faded away. No worsening of the symptoms with absence of any bone changes in the mandibular condyle and the temporal area was also confirmed in CBCT images of the TMJ.

\section{Case 2}

A 44-year-old female visited the Department of Oral Medicine with chief complaints of having spontaneous pain in both sides of the mandible and pain in the right side of the mandible when biting on the left side. The patient had a history of receiving treatments for myofascial pain 3 years ago, which went away after physical therapy, drug therapy, and botulinum toxin injection to reduce muscle hyperactivity. Recently, the upper left and lower left second molars were restored with gold inlays due to dental caries, and the upper left second molar received occlusal adjustment due to pain in the tooth when chewing. The symptoms initially improved, until 3 days later when the symptoms related to the chief complaints above developed. Clinical examination revealed $49 \mathrm{~mm}$ of active mouth opening, bilateral pain in the masseter muscle areas, and a clicking sound in the right TMJ area. The patient complained of bilateral tenderness in the TMJ and the masseter muscle areas upon palpation. The overjet and overbite were $1 \mathrm{~mm}$ each, and the midline was shifted to the left side by $1 \mathrm{~mm}$. Using an $8-\mu \mathrm{m}$ thick shimstock to study the intercuspal position showed occlusion of \#17, 15, 14 with \#44, 45, 47 and \#24 with \#35, 34. Panoramic radiograph showed no remarkable lesions in both TMJs, teeth, and the surrounding tissues. Transcranial radiographs showed no remarkable lesions in the mandibular condyles and no limitation of the anterior range of motion of the mandible. Lateral and postero-anterior cephalographs also did not reveal any notable lesions. Based on the results of the examinations, the patient was diagnosed with arthralgia of both TMJs and local myalgia of both masseter muscles. The patient received a similar set of treatments to the last case: physical therapy, self-treatment instruction, and medications including anti-inflammatory drugs (aceclofenac $100 \mathrm{mg} \mathrm{bid)}$ and tricyclic antidepressants (amitriptyline $10 \mathrm{mg} h s$ ).

The pain was relieved by the routine physical therapy and drug treatments, but after 2 months, the patient complained that the teeth were not occluding well after chewing food. Measuring the intercuspal position using an 8- $\mu \mathrm{m}$ thick shimstock showed that the occlusion on the right side was the same as before, but the teeth on the left side lost their contacts and were in infra-occlusion. Persistent muscle pain accompanied by change in occlusion was also observed. CBCT revealed flattening of the right mandibular condyle in the axial plane but stable cortical bone formation of the mandibular condyles. Osteoarthritis was not regarded as 
the main cause for the change in occlusion considering the absence of pain in the right TMJ and other clinical symptoms (Fig. 3). The occlusion on the left premolars was restored to the time of the first visit after continuous physical and drug therapies. The problem with the infra-occlusion on the left posterior area was referred to the Department of Prosthodontics, where prosthesis was made and temporarily cemented until the occlusion became stable and ready for permanent cementation.

The crowns on the lower left molars were permanently cemented after the symptoms were stabilized during the 6-month of monitoring period, but spontaneous pain recurred in both masseter muscle areas, and the patient reported occlusal instability. Bilateral tenderness was observed in the masseter muscle areas upon palpation, and a shimstock paper for measuring intercuspal position showed occlusion only on \#15, 14, and 24. The patient reported having to try very hard to chew and open mouth, which lead to using ultrasonography to evaluate her masseter muscle areas (Fig. 4). The results showed signs of atrophy at rest in both masseter muscles, and almost no contractility of both masseter muscles even during clenching. Muscle atrophy and muscle weakness were regarded as the main aggravating factors for the symptoms, and the patient was instructed to perform bilateral gum chewing in the posterior areas. In a follow-up visit 3 months after the continuous gum chewing, the patient reported decrease in pain in the masseter muscle areas and no fatigue or change in occlusion after chewing. Examining the intercuspal position showed occlusions of \#16, 15, 14 with 44, 45, 46 and \#24, 25, 27 with 37, 35, 34, and increased muscle contraction was also observed in both masseter muscle areas during ultrasonic examination (Fig. 4).

\section{DISCUSSION}

The clinical signs and symptoms of TMDs may include muscle dysfunction, functional disorders of the TMJ, and dysfunction in dentition, depending on the affected structures. Among them, muscle dysfunction may indicate pain, limited opening, and acute malocclusion. Appropriate diagnosis and treatment to reduce symptoms are required at an
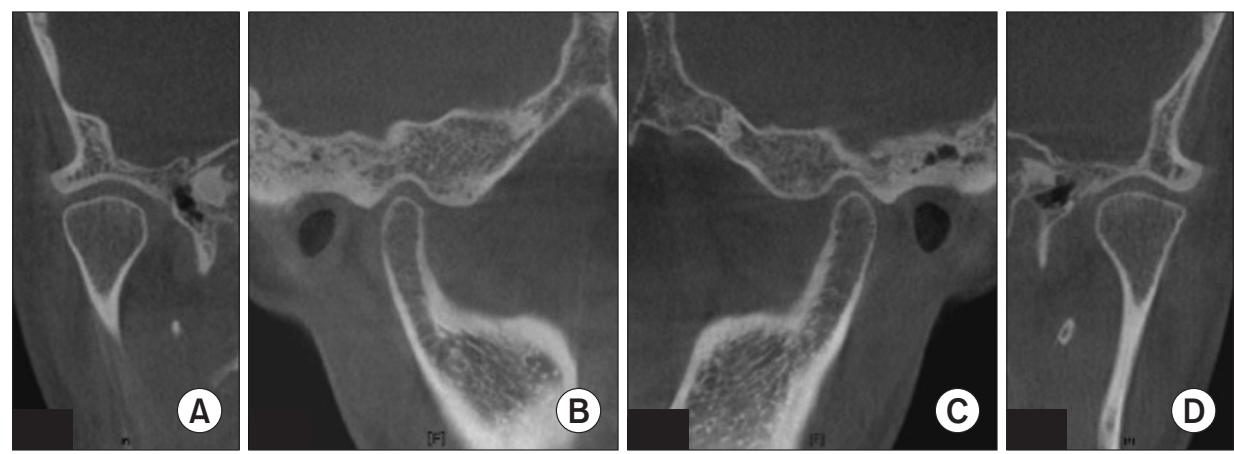

Fig. 3. Cone beam computed tomography image of case 2. (A) Right mandibular condyle in praracoronal plane, (B) Right mandibular condyle in parasagittal plane, (C) Left mandibular condyle in the parasagittal plane, (D) Left mandibular condyle in the paracoronal plane.
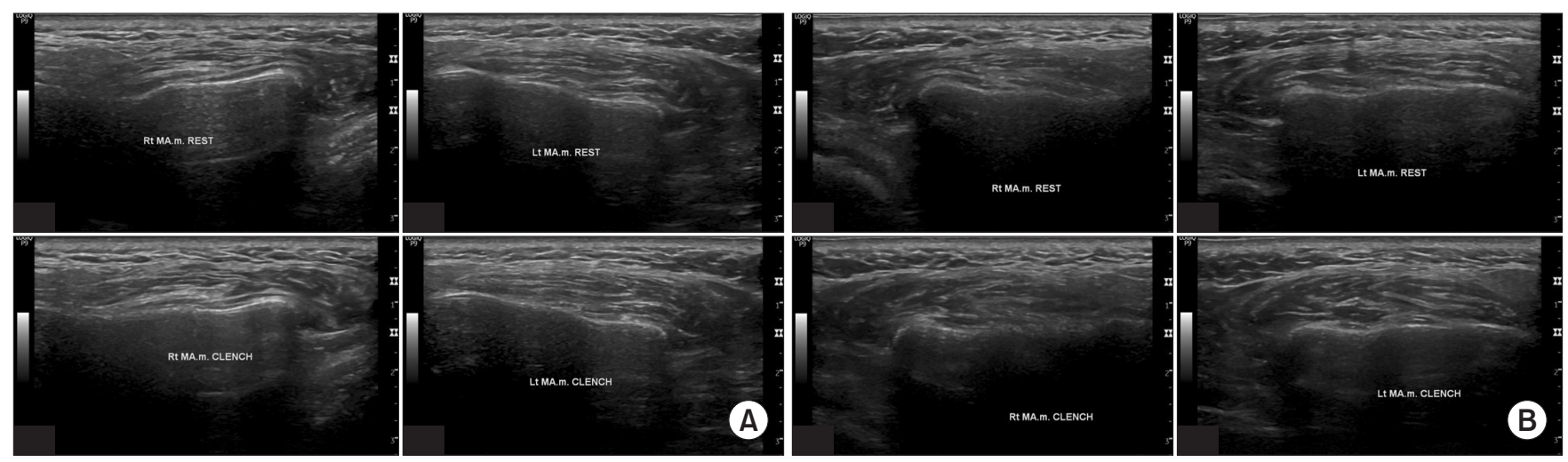

Fig. 4. Sonography before and after gum chewing exercise in case 2. (A) Before gum chewing, (B) 3 months after gum chewing. Rt, right; Ma. m, Masseter muscle; Lt, left. 
early stage, and treatment options usually focus on stabilizing the mandible and reducing muscle hyperactivity [2].

In these two cases, the patients were educated with home care therapy to reduce the contributing factors for muscle hyperactivity and excessive use of the mandible, but the symptoms did not improve with the conventional educational therapy. Both cases did not show any notable limitation in opening upon measurement. However, the patients reported difficulty in opening the mouth, with more force required to open to the same amount and showing decreased speed in opening. A study that used superficial electromyography to examine the relationship between TMDs and muscle fatigue found that the fatigue of the temporal and masseter muscles was directly related to severity of the symptoms of TMDs [3]. In a systematic review that evaluated persistent TMDs lasting for more than 3 months and musculoskeletal function, it was reported that persistent TMDs are related to musculoskeletal impairments such as reduced mouth opening, reduced maximal biting force and endurance, and reduced pressure threshold for sensing pain in the TMJ and masticatory muscles [1].

Both cases did not show any notable limitation in opening upon measurement. However, the patients reported difficulty in opening the mouth, with more force required to open to the same amount and showing decreased speed in opening. A study that used superficial electromyography to examine the relationship between TMDs and muscle fatigue found that the fatigue of the temporal and masseter muscles was directly related to severity of the symptoms of TMDs [3]. In a systematic review that evaluated persistent TMDs lasting for more than 3 months and musculoskeletal function, it was reported that persistent TMDs are related to musculoskeletal impairments such as reduced mouth opening, reduced maximal biting force and endurance, and reduced pressure threshold for sensing pain in the TMJ and masticatory muscles [1].

It is also noteworthy that the occlusal change occurred simultaneously as the TMD symptoms worsened. Generally, the association between TMDs and occlusal changes is mostly due to degenerative bone changes in the TMJ. However, the first case showed no degenerative bone changes or disc displacement in CT and MRI images, and while the second case showed degenerative bone changes in the TMJ, only flattening of the mandibular condyle took place with no inflammatory bone destruction such as erosion or destruction. Studies on muscle weakness and change in occlusion are very rare, with most of them related to botulinum toxin treatment or neuromuscular disease (e.g., Duchenne) [4,5]. It is therefore necessary to consider the association between TMDs and muscle weakness when patients with TMDs symptoms do not respond well to conventional conservative treatments, complain of a decreased speed and fatigue during opening and closing, and experience non-specific occlusal changes without degenerative osteoarthritis. Patients complaining of persistent TMDs are generally recommended to wear an occlusal appliance, but the patients in both cases did not want the oral appliance therapy with fear that their occlusion would worsen during the treatment.

To treat masticatory muscle weakness, the patients were instructed to perform gum chewing to achieve stable occlusion and muscle strengthening. This treatment approach may seem counterintuitive because chewing gum is generally regarded as an abnormal habit and a contributing factor of TMDs, and patients are discouraged from preferences that require excessive chewing such as gum or hard food until TMD related symptoms improve [6]. Research on chewing gums, however, reported that chewing gum for 40 to 60 minutes a day was not appropriate to become an experimental model for causing TMD pain [7]. In fact, one study reported that continuous gum chewing can be an effective exercise for the oromandibular function that does not accompany changes in mandibular angle or facial appearance [8]. Another study suggested that gum chewing can improve the anteroposterior occlusal balance and increase the occlusal contact area and occlusal force, thereby preventing downward rotation of the mandible [9]. In addition, rhythmic opening and closing of the mandible enable continuous force to be applied to the masticatory system, from which it was hypothesized that chewing gum could be used as a type of resistance exercise in the treatment of TMDs if the force was well controlled. A study on the effect of exercise therapy as part of the treatments for TMD reported that exercise education related to muscle endurance for 8 weeks resulted in improvement in pain, fatigue resistance, and muscle efficiency in patients with TMDs [10]. 
The gum chewing instruction given to the patients included placing an equal amount of gum on both molars at the same time to apply equal force to both sides of the posterior areas and chewing 1-2 times a day for about 15 minutes. Stabilization of occlusion and improvement of the pain symptoms were achieved in both cases, and increased contraction of the masseter muscles could be confirmed by the ultrasonic examination in the second case. Ultrasonography is a reliable tool for evaluating muscle strength [11]. To reduce errors during ultrasonography, the muscle strength was evaluated by measuring the volume of the masseter muscle by placing the ultrasound transducer on a horizontal plane in the middle part of the masseter muscle in the second case [12].

In treating TMDs, there are times when a patient is following treatment guidelines diligently only to find that symptoms get worse, which can be confusing for both the patient and the clinician. As presented in the cases above, non-specific occlusal changes, exacerbation of pain, and abnormal masticatory function may occur without degenerative changes in the TMJ. Muscle weakness from continuous decline of mandibular function may be responsible for these symptoms, for which exercise therapy may be considered. These cases may benefit from practicing gum chewing to achieve stable occlusion and muscle strengthening. However, to confirm the therapeutic effects of gum chewing as a viable treatment method for TMDs and to prove the relationship between muscle weakness and TMDs, more clinical cases need to be studied in the future. Comparing the effects of bilateral gum chewing with traditional resistance exercises and quantitative evaluation of muscle strength should be investigated as well.

If augmented with more research efforts as suggested above and with more positive results, it is expected that such efforts will make a meaningful contribution in the diagnosis and treatment of chronic TMDs.

\section{CONFLICT OF INTEREST}

No potential conflict of interest relevant to this article was reported.

\section{ORCID}

\author{
Ji Hoo Kim \\ https://orcid.org/0000-0002-0669-3309 \\ Hyun-Jeong Park \\ https://orcid.org/0000-0002-5237-005X \\ Ji-Won Ryu \\ https://orcid.org/0000-0002-5586-8195
}

\section{REFERENCES}

1. Dinsdale A, Liang Z, Thomas L, Treleaven J. Are jaw range of motion, muscle function and proprioception impaired in adults with persistent temporomandibular disorders? A systematic review and meta-analysis. J Oral Rehabil 2020;47:1448-1478.

2. Okeson JP. Management of temporomandibular disorders and occlusion. 7th ed. St. Louis: Elsevier; 2013.

3. Woźniak K, Lipski M, Lichota D, Szyszka-Sommerfeld L. Muscle fatigue in the temporal and masseter muscles in patients with temporomandibular dysfunction. Biomed Res Int 2015;2015:269734.

4. Ryu JW. Case report: anterior open bite after injection of botulinum toxin on masseter muscles. J Oral Med Pain 2013;38:325331.

5. van den Engel-Hoek L, de Groot IJ, Sie LT, et al. Dystrophic changes in masticatory muscles related chewing problems and malocclusions in Duchenne muscular dystrophy. Neuromuscul Disord 2016;26:354-360.

6. Correia D, Real Dias MC, Castanho Moacho A, et al. An association between temporomandibular disorder and gum chewing. Gen Dent 2014;62:e33-e36.

7. Al Sayegh S, Vasilatou I, Kumar A, et al. Experimental pain and fatigue induced by excessive chewing. BMC Oral Health 2020;20:179.

8. Kim JH, Park HS, Kim MY, Kim KW. Effect of gum-chewing on facial appearance and stomatognathic system. J Korean Dent Sci 2014;7:16-24.

9. Masumoto N, Yamaguchi K, Fujimoto S. Daily chewing gum exercise for stabilizing the vertical occlusion. J Oral Rehabil 2009;36:857-863.

10. Barbosa MA, Tahara AK, Ferreira IC, Intelangelo L, Barbosa AC. Effects of 8 weeks of masticatory muscles focused endurance exercises on women with oro-facial pain and temporomandibular disorders: a placebo randomised controlled trial. J Oral Rehabil 2019;46:885-894

11. Reis Durão AP, Morosolli A, Brown J, Jacobs R. Masseter muscle measurement performed by ultrasound: a systematic review. Dentomaxillofac Radiol 2017;46:20170052.

12. Chang PH, Chen YJ, Chang KV, Wu WT, Özçakar L. Ultrasound measurements of superficial and deep masticatory muscles in various postures: reliability and influencers. Sci Rep 2020;10:14357. 